\title{
Differential expression of CD34 in cancers of the breast.
}

Shahan Mamoor, MS1

1shahanmamoor@gmail.com

East Islip, NY 11730

Breast cancer affects women at relatively high frequency ${ }^{1}$. We mined published microarray datasets 2,3 to determine in an unbiased fashion and at the systems level genes most differentially expressed in the primary tumors of patients with breast cancer. We report here significant differential expression of CD34 when comparing primary tumors of the breast to the tissue of origin, the normal breast. CD34 expression in primary tumors of the breast was significantly lower than in normal breast tissue, and relapse-free and overall survival in breast cancer patients was significantly worse in patients with low tumor expression of CD34 than in patients with high tumor expression of CD34. CD34 may be of relevance to initiation, maintenance or progression of cancers of the female breast.

Keywords: breast cancer, CD34, systems biology of breast cancer, targeted therapeutics in breast cancer. 
Invasive breast cancer is diagnosed in over a quarter of a million women in the United States each year ${ }^{1}$ and in 2018 , breast cancer was the leading cause of cancer death in women worldwide ${ }^{4}$. While patients with localized breast cancer are provided a 99\% 5-year survival rate patients with regional breast cancer, cancer that has spread to lymph nodes or nearby structures, are provided an $86 \% 5$-year survival rate 5,6 . Patients with metastasis to distant sites are provided a $27 \% 5$-year survival rate 5,6 . Understanding how primary tumors are most transcriptionally different from the tissue from which they originate, the breast, can facilitate development of novel diagnostic and therapeutics to promote early detection and enhanced treatment, and contribute to efforts to prevent progression to metastatic stages. We mined published microarray data 2,3 to understand at the transcriptome level and in an unbiased fashion genes most differentially expressed in primary tumors of the breast as compared to normal breast tissue. CD34 emerged as among the most differentially expressed genes in cancer of the female breast.

\section{Methods}

We utilized datasets GSE124648² and GSE109169³ and for this differential gene expression analysis of female breast cancer. GSE124648 was generated using Affymetrix Human Genome U133A Array technology with $n=10$ normal breast tissue and $n=126$ tumors of the breast and; analysis was performed using platform GPL96. GSE109169 was generated using Affymetrix Human Exon 1.0 ST Array technology with $n=25$ normal breast tissue and $n=25$ tumors of the breast and; analysis was performed using platform GPL5175. The tissues from this first dataset are paired tissues (25 tumors matching 25 breast tissues from 25 patients). The Benjamini and Hochberg method of $p$-value adjustment was used for ranking of differential expression but raw $p$-values were used to assess statistical significance of global differential 
expression. Log-transformation of data was auto-detected, and the NCBI generated category of platform annotation was used. A statistical test was performed to evaluate whether CD34 expression was significantly between primary breast tumors and breast tissue using a twotailed, unpaired t-test with Welch's correction. We used PRISM for all statistical analyses of differential gene expression in human breast cancer (Version 8.4.0)(455). For Kaplan-Meier survival analysis, we used the Kaplan-Meier plotter online tool7 for correlation of CD34 mRNA expression levels with relapse-free survival (RFS) in $n=3951$ breast cancer patients and with overall survival (OS) in $n=1402$ breast cancer patients.

\section{Results}

We performed discovery of genes associated with breast cancer in females by mining two independently published microarray datasets 2,3 .

\section{CD34 is differentially expressed in primary tumors of the breast.}

Comparison of 126 tumors of the breast to 10 normal breast tissues revealed that the gene CD34 was among the genes most differentially expressed in tumors of the breast in female breast cancer (Table 1). When sorting each of the genes expressed in primary breast tumors based on significance of difference as compared to normal breast tissue, CD34 ranked 150 out of 22283 total transcripts, equating to $99.3 \%$ differential expression (Table 1). Differential expression of CD34 in female breast cancer was statistically significant (Table 1; $p=1.8 \mathrm{E}-15)^{2}$

Analysis of a second microarray dataset ${ }^{3}$, here comparing 25 tumors of the breast to 25 normal breast tissues again revealed significant differential expression of CD34 in tumors of the breast in female breast cancer (Table 2). When sorting each of the genes expressed in 
tumors of the breast based on significance of difference as compared to normal breast tissue, CD34 ranked 91 out of 19076 total transcripts, equating to $99.5 \%$ differential expression (Table 2). Differential expression of CD34 in female breast cancer was again statistically significant (Table 2; $p=6.27 \mathrm{E}-15)$.

CD34 is expressed at significantly lower levels in breast tumors as compared to the breast.

We obtained exact mRNA expression levels for CD34 from the breast and from breast tumors to understand the magnitude and direction of CD34 expression change in human breast cancer. In both datasets, CD34 was expressed at lower levels in tumors of the breast as compared to normal breast tissue (Figure 1). Decreased expression of CD34 in primary breast tumors was statistically significant (Figure 1: $p<0.0001$ and Figure 2: $p<0.0001$ ). We calculated a mean fold change of $0.8771 \pm 0.0405$ and $0.8593 \pm 0.0388$ when comparing primary tumors of the breast to normal breast tissues (Table 1 and Table 2, respectively).

\section{CD34 expression associates with survival outcomes in breast cancer.}

We performed Kaplan-Meier survival analysis in an attempt to correlate CD34 mRNA expression levels with survival outcomes in 3951 breast cancer patients. We found a statistically significant correlation between CD34 expression and relapse-free survival (RFS) in patients with breast cancer (Figure 3; log rank p-value: 4e-16 for relapse-free survival, hazard ratio: 0.63 (0.57-0.71) (Fig. 3)). Low CD34 mRNA levels were a negative prognostic indicator in patients with breast cancer. Median RFS was 35 months for patients with low tumor expression of CD34 while median RFS was 76.02 months for patients with high tumor expression of CD34 (Table 3).

4 OF 17 
We also evaluated whether CD34 mRNA expression was correlated with overall survival in 1402 breast cancer patients. We found a statistically significant correlation between CD34 expression and overall survival (OS) in patients with breast cancer (Figure 4; log rank $p$-value: 0.027 for overall survival, hazard ratio: 0.78 (0.63-0.97) (Fig. 4)). Low CD34 mRNA levels were again a negative prognostic indicator in patients with breast cancer. Median OS was 77.4 months for patients with low tumor expression of CD34 while median OS was 135.88 months for patients with high tumor expression of CD34 (Table 4).

Thus, through comparative transcriptome analysis of primary tumors of the breast and normal breast tissue, we found that differential expression and down-regulation of CD34 was among the most significant transcriptional features in primary tumors from patients with breast cancer. CD34 expression in primary tumors of the breast demonstrated a significant correlation with both relapse-free and overall survival in patients with breast cancer, with high expression of CD34 correlating with improved survival.

\section{Discussion}

Expression of CD34, an 110 kilodalton glycoprotein was described at the protein level in hematopoietic progenitors, vascular endothelium, capillaries of most tissues and the umbilical artery. Interestingly, though endothelial cells proliferation in culture expressed CD34 RNA, the antibodies utilized by investigators in one study did not detect protein-level CD34 expression. While endothelial cell contacts with tight junctions were devoid of CD34 protein staining, CD34 expression was observed at membrane processes at adjacent endothelial cells ${ }^{8}$. Expression of CD34 has also been observed in the sinusoidal cells of the fetal liver, the fetal bone marrow, in the yolk sac, in endothelial cells of the adult lung and kidney and in cells of the nervous systems including the embryonic neural tube ${ }^{9}$. CD34 is important as a marker of cells in the 
hematopoietic system, and its expression or lack thereof can signify functionality of hematopoietic stem and progenitor cells. Transplantation of mice with CD34- bone marrow cells gives rise to CD34+ progenitors with long-term engraftment potential in secondary transplantation hosts ${ }^{10}$. Transplantation of a single CD34lo/-, c-kit+ Sca-1+ cell negative for lineage markers (Lin-) was capable of reconstituting lymphohematopoietic cells in $21 \%$ of recipients ${ }^{11}$. CD34 expressed on high endothelial venules can interact with L-selectin on leukocytes to facilitate lymphocyte migration across the endothelium ${ }^{12}$. Erythroid and myeloid differentiation from CD34-deficient embryonic stem cells in vitro is delayed, demonstrating that CD34 can support commitment to the erythroid and myeloid lineages, and colony formation potential for these lineages in the yolk sacs and fetal livers of CD34-deficient embryos is reduced $^{13}$. While adult peripheral blood is apparently normal in CD34-deficient mice, differentiation of progenitors isolated from CD34-deficient mice in liquid culture in vitro is deficient ${ }^{13}$.

CD34 has been described as a marker of tumor lymphatic endothelial cells in human cancers $^{14}$, and perturbed expression of CD34 has been described by multiple studies in human cancers including gastric cancer ${ }^{15}$, small cell lung cancer ${ }^{16}$, and breast cancer ${ }^{17-19}$. In gastric cancer, CD34 expression used a surrogate for microvessel density was correlated with histological grade ${ }^{15}$. Two out of eleven small-cell lung cancer cell lines were positive for expression of CD3416. One study found that fibroblasts around invasive breast carcinomas were CD34 positive and negative for smooth muscle actin (SMA); while fibroblasts around normal duct-lobule units were also CD34 positive and negative for SMA, fibroadenoma fibroblasts were CD34 positive and SMA positive ${ }^{17}$. In ductal carcinoma in situ, loss of CD34 appeared to be more frequent in high grade tumors than in low and intermediate grade 
tumors ${ }^{17}$. Microvessel density, or Chalkley count, has been proposed as a means of evaluating angiogenesis by immunohistochemical means in tumor tissues ${ }^{18}$, and one study presented a promising immunohistochemical method of CD34 evaluation in tumor tissues from patients with breast cancer ${ }^{19}$. Finally, one study demonstrated that changes in microvessel density as assessed by CD34 staining could be observed in tumor tissue from patients with breast cancer; while the authors argued that these changes were not biologically important because they were small, the change in microvessel density displayed a trend towards correlation with patient HER2 status. Thus, while it has been argued that solid tumors save for endothelial cancers generally do not express CD3416, CD34 expression has been demonstrated in multiple cancers including human breast cancer and investigators have attempted to measure CD34 expression in patient tumors by immunohistochemical methods as a quantitative indicator of tumor microvessel density.

We found that CD34 was among the genes most differentially expressed transcriptomewide in human breast cancer when comparing primary tumors of the breast to the tissue of origin, normal breast tissue. CD34 was expressed at significantly lower levels in primary breast tumors than in the breast, and high tumor expression of CD34 was associated with enhanced relapse-free and overall survival in patients with breast cancer. CD34 may be relevant to the initiation or progression of human breast cancer. 


\section{References}

1. DeSantis, C.E., Ma, J., Goding Sauer, A., Newman, L.A. and Jemal, A., 2017. Breast cancer statistics, 2017, racial disparity in mortality by state. CA: a cancer journal for clinicians, 67(6), pp.439-448.

2. Sinn, B.V., Fu, C., Lau, R., Litton, J., Tsai, T.H., Murthy, R., Tam, A., Andreopoulou, E., Gong, Y., Murthy, R. and Gould, R., 2019. SET ER/PR: a robust 18-gene predictor for sensitivity to endocrine therapy for metastatic breast cancer. NPJ breast cancer, 5(1), pp. $1-8$.

3. Chang, J.W., Kuo, W.H., Lin, C.M., Chen, W.L., Chan, S.H., Chiu, M.F., Chang, I.S., Jiang, S.S., Tsai, F.Y., Chen, C.H. and Huang, P.H., 2018. Wild-type p53 upregulates an early onset breast cancer-associated gene GAS7 to suppress metastasis via GAS7-CYFIP1mediated signaling pathway. Oncogene, 37(30), pp.4137-4150.

4. Bray, F., Ferlay, J., Soerjomataram, I., Siegel, R.L., Torre, L.A. and Jemal, A., 2018. Global cancer statistics 2018: GLOBOCAN estimates of incidence and mortality worldwide for 36 cancers in 185 countries. CA: a cancer journal for clinicians, 68(6), pp.394-424.

5. ACS Cancer Facts \& Figures 2019. https://www.cancer.net/cancer-types/breast-cancermetastatic/statistics.

6. Survival Rates for Breast Cancer. https://www.cancer.org/cancer/breast-cancer/ understanding-a-breast-cancer-diagnosis/breast-cancer-survival-rates.html

7. Györffy, B., Lanczky, A., Eklund, A.C., Denkert, C., Budczies, J., Li, Q. and Szallasi, Z., 2010. An online survival analysis tool to rapidly assess the effect of 22,277 genes on breast cancer prognosis using microarray data of 1,809 patients. Breast cancer research and treatment, 123(3), pp.725-731.

8. Fina, L., Molgaard, H.V., Robertson, D., Bradley, N.J., Monaghan, P., Delia, D., Sutherland, D.R., Baker, M.A. and Greaves, M.F., 1990. Expression of the CD34 gene in vascular endothelial cells.

9. Lin, G., Finger, E. and Gutierrez-Ramos, J.C., 1995. Expression of CD34 in endothelial cells, hematopoietic progenitors and nervous cells in fetal and adult mouse tissues. European journal of immunology, 25(6), pp.1508-1516.

10. Zanjani, E.D., Almeida-Porada, G., Livingston, A.G., Flake, A.W. and Ogawa, M., 1998. Human bone marrow CD34-cells engraft in vivo and undergo multilineage expression that includes giving rise to CD34+ cells. Experimental hematology, 26(4), pp.353-360.

8 OF 17 
11. Osawa, M., Hanada, K.I., Hamada, H. and Nakauchi, H., 1996. Long-term lymphohematopoietic reconstitution by a single CD34-low/negative hematopoietic stem cell. Science, 273(5272), pp.242-245.

12. Baumheter, S., Singer, M.S., Henzel, W., Hemmerich, S., Renz, M., Rosen, S.D. and Lasky, L.A., 1993. Binding of L-selectin to the vascular sialomucin CD34. Science, 262(5132), pp. 436-438.

13. Cheng, J., Baumhueter, S., Cacalano, G., Carver-Moore, K., Thibodeaux, H., Thomas, R., Broxmeyer, H.E., Cooper, S., Hague, N., Moore, M. and Lasky, L.A., 1996. Hematopoietic defects in mice lacking the sialomucin CD34.

14. Fiedler, U., Christian, S., Koidl, S., Kerjaschki, D., Emmett, M.S., Bates, D.O., Christofori, G. and Augustin, H.G., 2006. The sialomucin CD34 is a marker of lymphatic endothelial cells in human tumors. The American journal of pathology, 168(3), pp.1045-1053.

15. Tenderenda, M., Rutkowski, P., Jesionek-Kupnicka, D. and Kubiak, R., 2001. Expression of CD34 in gastric cancer and its correlation with histology, stage, proliferation activity, p53 expression and apoptotic index. Pathology Oncology Research, 7(2), pp.129-134.

16. Yamane, H., Kiura, K., Tabata, M., Bessho, A., Tsuchida, T., Motoda, K., Hiraki, A., Ueoka, H. and Harada, M., 1997. Small cell lung cancer can express CD34 antigen. Anticancer research, 17(5 A), pp.3627-3632.

17. Chauhan, H., Abraham, A., Phillips, J.R.A., Pringle, J.H., Walker, R.A. and Jones, J.L., 2003. There is more than one kind of myofibroblast: analysis of CD34 expression in benign, in situ, and invasive breast lesions. Journal of clinical pathology, 56(4), pp.271-276.

18. Offersen, B.V., Borre, M. and Overgaard, J., 2003. Quantification of angiogenesis as a prognostic marker in human carcinomas: a critical evaluation of histopathological methods for estimation of vascular density. European Journal of Cancer, 39(7), pp.881-890.

19. Mikalsen, L.T.G., Dhakal, H.P., BRULAND, Ø.S., Nesland, J.M. and Olsen, D.R., 2011. Quantification of angiogenesis in breast cancer by automated vessel identification in CD34 immunohistochemical sections. Anticancer research, 31(12), pp.4053-4060.

20. Bottini, A., Berruti, A., Bersiga, A., Brizzi, M.P., Allevi, G., Bolsi, G., Aguggini, S., Brunelli, A., Betri, E., Generali, D. and Scaratti, L., 2002. Changes in microvessel density as assessed by CD34 antibodies after primary chemotherapy in human breast cancer. Clinical cancer research, 8(6), pp.1816-1821. 


\section{Table 1: CD34 is differentially expressed in human breast cancer.}

Rank of differential expression, probe ID, p-value of global differential expression, $t, a$ moderated t-statistic, B, the log-odds of differential expression between the groups compared, fold change of CD34 in primary breast tumors as compared to normal breast, gene and gene name are listed in this chart.

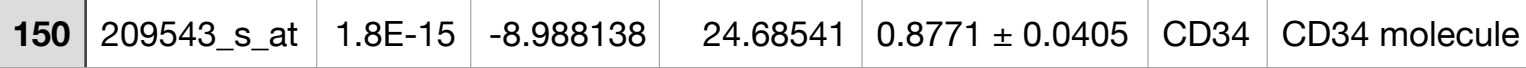




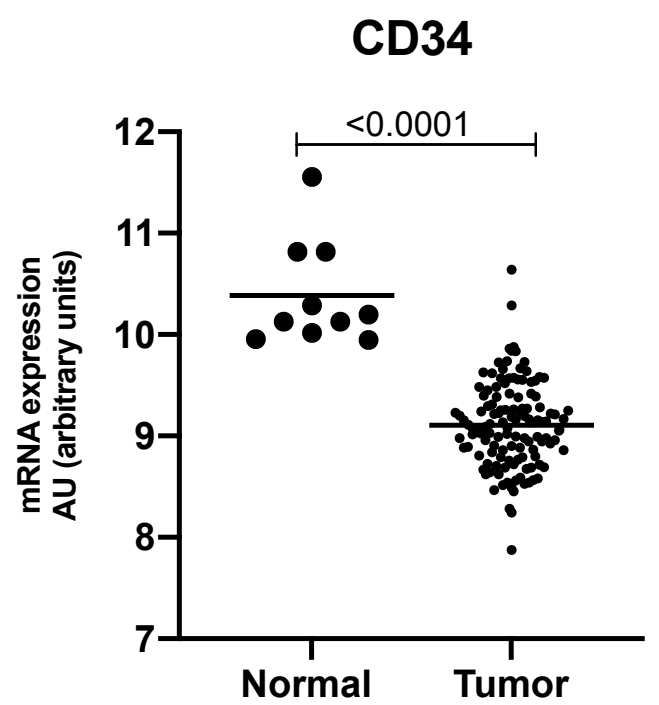

Figure 1: CD34 is expressed at significantly lower levels in primary breast tumors as compared to normal breast tissue.

The mRNA expression level of CD34 in normal breast tissue (left) and in primary tumors of the breast (right) is graphically depicted with the result of a statistical test evaluating significance of difference in CD34 expression between normal breast tissue and primary tumors of the breast, a $p$-value, listed above. 


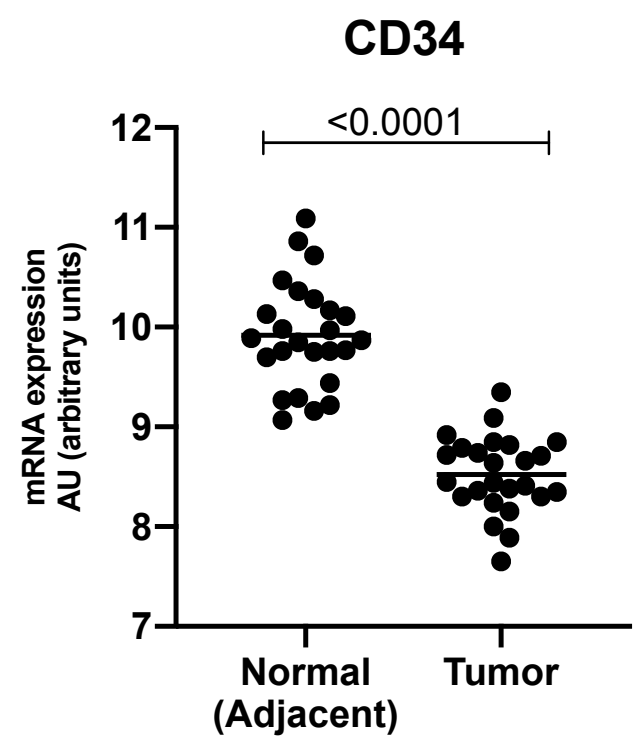

Figure 2: CD34 is expressed at significantly lower levels in primary breast tumors as compared to normal breast tissue.

The mRNA expression level of CD34 in normal breast tissue (left) and in primary tumors of the breast (right) is graphically depicted with the result of a statistical test evaluating significance of difference in CD34 expression between normal breast tissue and primary tumors of the breast, a $p$-value, listed above. 


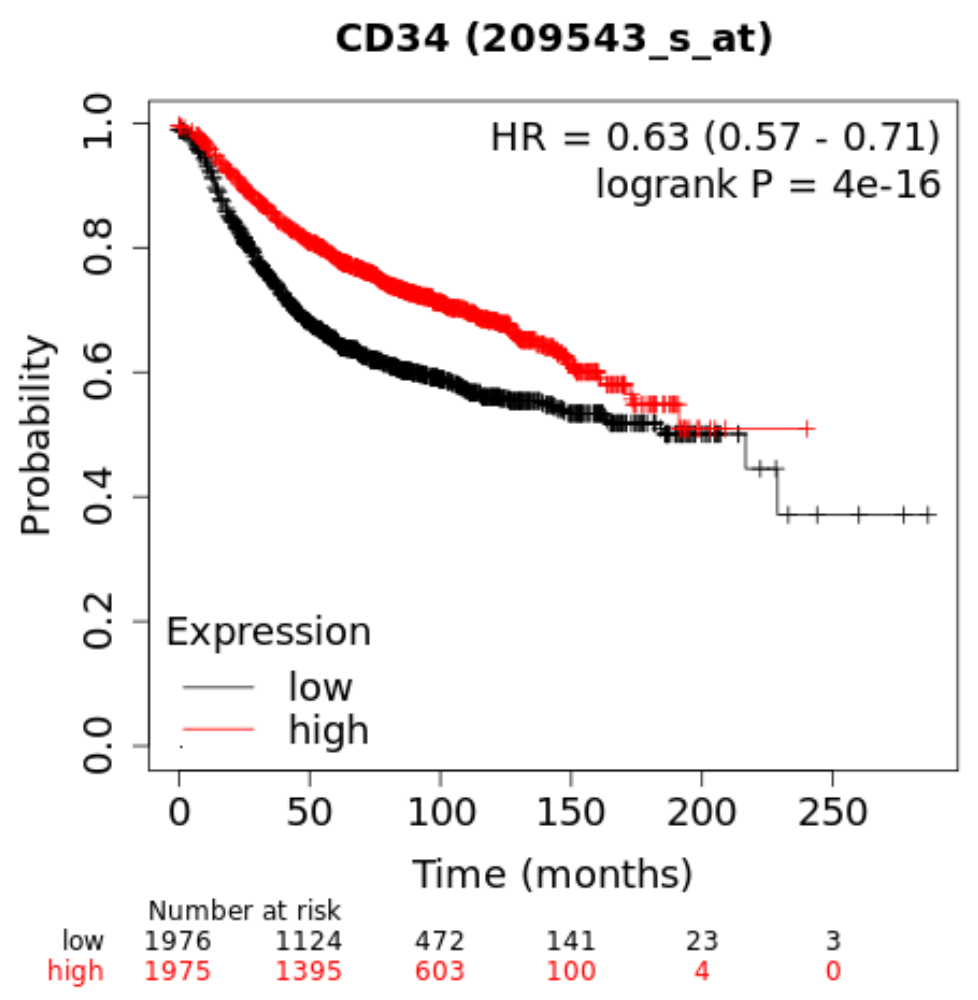

Figure 3: CD34 expression correlates with relapse-free survival in patients with breast cancer.

Depicted in this Kaplan-Meier plot is the probability of relapse-free survival (RFS) for $n=3951$ total patients stratified into two groups, based on low or high expression of CD34 in patient primary tumors. The log rank $p$-value denoting statistical significance of difference in distant relapse-free survival when comparing the two groups, as well as hazard ratio for this comparison is listed above. Listed below is the number of patients at risk (number of patients alive) per interval, after stratification based on CD34 expression; in the first interval, number at risk is number of patients alive; in each subsequent interval, number at risk is the number at risk less those who have expired or are censored. 
The relapse-free survival of $n=3951$ breast cancer patients based on stratification into low or high tumor expression of CD34 is listed in this chart.

Table 3: Median relapse-free survival is greater in breast cancer patients with high primary tumor expression of CD34. 


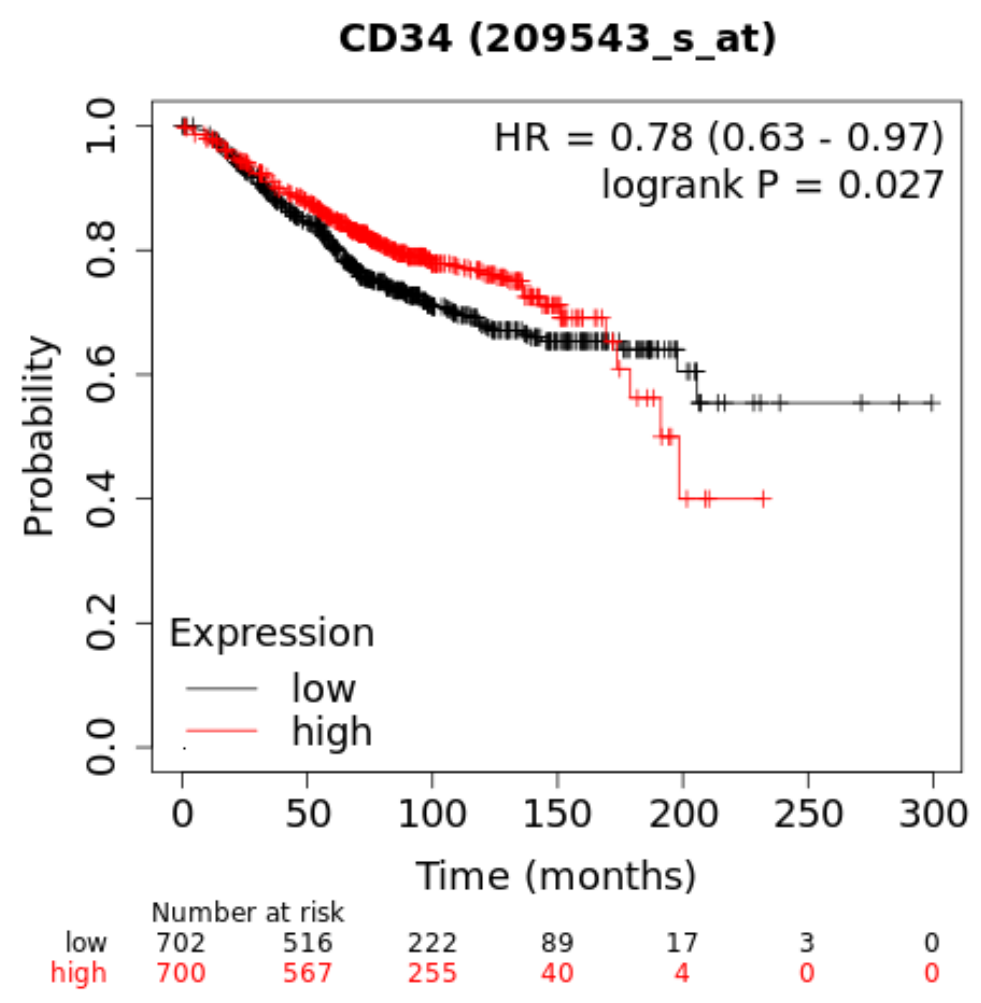

Figure 4: CD34 expression correlates with overall survival in patients with breast cancer.

Depicted in this Kaplan-Meier plot is the probability of overall survival (OS) for $n=1402$ total patients stratified into two groups, based on low or high expression of CD34 in patient primary tumors. The log rank $p$-value denoting statistical significance of difference in distant relapsefree survival when comparing the two groups, as well as hazard ratio for this comparison is listed above. Listed below is the number of patients at risk (number of patients alive) per interval, after stratification based on CD34 expression; in the first interval, number at risk is number of patients alive; in each subsequent interval, number at risk is the number at risk less those who have expired or are censored. 
